\title{
Lecture mythocritique des Faux-Monnayeurs d'André Gide
}

\author{
David KEYPOUR \\ Huron College
}

Le propos de cette étude est d'examiner tout d'abord l'interprétation que Gide donne de la parabole évangélique de l'Enfant Prodigue, et de voir ensuite le fonctionnement du mythe personnel ainsi formé dans la structuration de l'oeuvre narrative la plus complexe que Gide ait écrite environ 20 ans plus tard, les Faux-Monnayeurs. Pour éclairer ce rapprochement, appel sera fait au manuscrit partiel de ce roman. ${ }^{1}$ L'approche s'apparente donc à la psychocritque de Mauron ${ }^{2}$ tandis que la conclusion ouvrira une perspective de critique féministe.

Il est à remarquer que, comme plusieurs des écrits de Gide, sa version de la parabole évangélique, publiée en 1907 sous le titre Le Retour de l'Enfant Prodigue et classée comme "traité," est mise sous le signe explicite du "retour," et que, dans les dialogues qui la composent, les deux autres volets du "triptyque," à savoir, le "départ" et le "voyage" proprement dit, sont très succinctement évoqués, chaque fois d'une façon différente et de plus en plus allusive, de sorte que c'est vraiment le retour qui constitue l'événement, comme si le retour était inscrit d'avance dans le destin du Prodigue.

Mais ce retour, bien qu'il semble signifier un échec, n'est pas totalement négatif. Le Prodigue a quand même fait l'expérience de la liberté, a découvert le monde extérieur, a compris ce dont il était capable et ses limites avant de se faire "mercenaire" et de retourner au bercail. Dans ce sens l'oeuvre, mise à part sa signification 
religieuse, est le condensé d'un roman d'apprentissage. Il est positif aussi parce que ce retour est un terme nécessaire au destin du Prodigue, un terme sans lequel son destin n'aurait pas de sens, sans lequel son destin serait inachevé et absurde. Comparé au voyage imaginaire ou mensonger d'Urien et au départ impossible de Tityre de Paludes, au désarroi et à l'impuissance finale de Michel de L'Immoraliste, à l'exil que le pasteur de la Symphonie impose à son fils et à la mort que Thésée apporte à son père en retournant en Attique, le retour du Prodigue ressemble à la dernière section d'une courbe qui vient compléter un cercle parfait. C'est que son départ a été un ébranlement de l'ordre dans la maison, son absence a déséquilibré l'architecture de la famille. Les bras ouverts du père, l'attente épleurée de la mère signifiaient jusqu'à ce jour le vide qui vient d'être comblé. Ce retour était dans l'ordre des choses, et désormais tout est en ordre. C'est donc un retour heureux, et non pas seulement parce qu'il donne cohérence au destin personnel du Prodigue.

L'opportunité du retour du Prodigue est de plus mise en évidence par le fait qu'il prélude au départ de son puîné. Tout se passe comme si le destin du Prodigue était lié à celui de son jeune frère, comme s'il fallait qu'il parte et qu'il revienne pour que le plus jeune puisse trouver le courage de quitter à son tour la maison paternelle. C'est cet enchaînement et l'encouragement que le Prodigue apporte à son puîné qui scandalisèrent les bien-pensants catholiques, dont Claudel, lorsque parut ce traité.

Or ce qu'il fallait reprocher à Gide, si reproche est de mise, c'est d'avoir enrichi la famille du Prodigue d'un troisième fils qui n'existe pas dans l'originale évangélique. Car que sert de l'avoir inventé s'il ne devait pas avoir un rôle à jouer? Et quel rôle autre que celui de l'émule de son aîné? Et si le destin du Prodigue est un destin heureux, c'est parce qu'il s'inscrit dans le cadre d'une famille telle que son retour puisse devenir un nouveau point de départ, l'occasion d'une nouvelle libération. L'existence du puîné subvertit la signification de la parabole évangélique parce qu'elle enlève au retour du Prodigue le caractère d'un repentir et transforme sa vocation en celle d'un éveilleur, celle précisément que Gide se reconnaissait contre toutes accusations. L'invention du puîné est donc une modification à la fois structurelle et signifiante que Gide apporte à la parabole pour en faire son mythe personnel.

Mais par ailleurs, le Prodigue n'est prodigue que parce qu'il a un frère aîné, un frère qui veille comme un gardien plus jaloux que le père sur l'héritage paternel et dont l'ombre protectrice semble avoir préservé le Prodigue lui-même de la perdition à laquelle il s'était risqué en reniant cet héritage. C'est dire que la condition première 
de l'équilibre est un ordre familial où la mort du père, inévitable par nature, est déjà compensée par la présence d'un substitut, d'un frère aîné qui assure la pérennité de l'ordre dès avant la disparition du père. C'est donc autant l'autorité du père que le privilège de l'aîné que le Prodigue rejette en partant. Mais, que la présence de l'aîné vienne à manquer, il n'y aurait point de Prodigue à proprement parler, et la menace de la perdition pèserait sur la vie de tout autre enfant de la famille.

Cette structure familiale établie comme norme dans le mythe personnel que Gide se construit à partir de la parabole biblique permet de classer les familles qui peuplent le roman des Faux-Monnayeurs selon leur degré de ressemblance au modèle et d'éclairer d'un nouveau jour le destin de certains personnages.

Des cinq familles principales, celle de La Pérouse est la plus éloignée de l'archétype. Son petit fils Boris n'a ni père, ni frères; menacé de toutes parts comme une proie sans défense, il succombe à des troubles émotifs et meurt de la façon la plus tragiquement absurde.

La famille du pasteur Vedel n'a pas de benjamin et Alexandre le fils aîné a déserté son rôle de protecteur pour aller vivre en Afrique. La famille ainsi déséquilibrée, Armand qui aurait pu en être le Prodigue est embourbé parmi ses soeurs, ${ }^{3}$ remâchant son désespoir qu'il essaie de cacher sous un cynisme de façade. Il n'aura jamais la force de quitter sa famille qui sombre dans le malheur et la faillite financière.

A la famille de Passavant il manque le fils médiateur, celui qui aurait pu jouer le rôle du Prodigue. Le cadet Gontran demeure coupé de Robert à cause de ce hiatus structurel, c'est pourquoi il est condamné à vivoter à la pension Azaïs, privé d'appui fraternel et d'exemple à suivre.

La famille Molinier est composée conformément à l'archétype; mais Molinier a abdiqué son autorité paternelle et Vincent le fils aîné loin de rester pour assurer la permanence de l'ordre s'est laissé débaucher par Lilian, donc le désordre atteint également ses frères. Olivier qui aurait pu être le fils prodigue est en passe de se dévoyer en compagnie de Robert; Georges vole, écoule de la fausse monnaie et participe au crime contre Boris. La famille complètement désintégrée ne tient que par la mère. Or il est significatif que la mère se déclare impuissante à exercer la moindre autorité sur les enfants et qu'elle supplie son frère l'oncle Edouard de remplir la place désertée par le père et le fils aîné. Dès lors, Edouard se trouve habilité à les figurer tous deux à la 
fois, et à partir du moment où Olivier se met sous sa protection, Georges aussi s'amende.

Strouvilhou, Cob-Lafleur et Ghéridanisol semblent former un trio hiérarchisé selon le même ordre que celui des trois frères du modèle mythique. Mais étant sans famille et hétéroclytes, chacun représente l'individu le plus éloigné de la place qu'il aurait pu occuper dans la famille archétypale. Ainsi, ce trio figure le modèle diamétralement opposé au modèle familial du Prodigue. C'est pourquoi, au lieu que leur association leur apporte l'ordre et le dynamisme libérateur inhérents au modèle mythique, elle aboutit à multiplier le vice et le crime. Ce trio est l'incarnation du mal irréparable.

La famille Profitendieu est la seule qui soit à la fois conforme à la structure de la famille archétypale et dont les membres ne dévient pas du rôle imparti à chacun. Le père est présent et imbu de son autorité, Charles le fils aîné est soumis au père et le seconde de tout son zèle, Bernard joue son rôle de prodigue en abondonnant le foyer, et le cadet Caloub veille dans l'ombre en attendant que son heure arrive de suivre l'exemple de Bernard. Le parallélisme entre Caloub et le puîné de l'Enfant Prodigue est parfaitement suggéré par l'équivalence entre la fin du journal d'Edouard qui est aussi la fin des Faux-Monnayeurs et l'épilogue du Retour de l'Enfant Prodigue. L'on sait en effet que cet épilogue consiste en un dialogue entre le Prodigue et son puîné au cours duquel on prend acte une dernière fois du retour du Prodigue et où l'on assiste au départ du puîné sous l'égide de ce dernier. De même Edouard, après avoir rapporté dans son journal la rencontre entre le petit Caloub et Bernard, enregistre le retour de ce dernier et ajoute: "Je suis bien curieux de connaitre Caloub." Cette connaissance du jeune personnage par le romancier, son entrée prévue dans la sphère de l'aventure romanesque signifie sans doute l'ouverture d'une nouvelle carrière, le départ d'un nouveau Prodigue.

Et ce qui confirme le dessein profond ou inconscient de concevoir cette famille suivant le modèle de la famille du Prodigue est un long développement du chapitre II des Faux-Monnayeurs que Gide a en partie éliminé de la version définitive, développement qui se trouve dans le manuscrit partiel mentionné ci-dessus.

Dans ce manuscrit comme dans la version définitive, le chapitre II commence par la rentrée chez soi de Profitendieu en compagnie d'Oscar Molinier. On se souvient qu'ils remontent ensemble le boulevard Saint-Germain et qu'ils discutent de la 
manière dont il convient de mettre fin aux agissements d'une bande de jeunes garçons dévoyés par des prostituées. Les deux hommes se séparent; Profitendieu peut enfin presser le pas, et sitôt chez lui, il se coule un bain. Jusqu'ici, le manuscrit est très proche du texte du roman. A partir d'ici, les feuillets sont surchargés d'ajouts, et on distingue trois états successifs du passage où Antoine avise Profitendieu du départ de Bernard. Dans le premier état, aucune mention n'est faite de la lettre que Bernard a laissée à l'intention de son père. Le troisième état diffère peu du texte définitif. Or à cet endroit est intercalé un feuillet de papier différent des autres et qui contient la lettre de Bernard. Il est clair qu'en un second temps cette lettre a été ajoutée au premier jet et que des retouches sont venues l'y intégrer. Cette lettre est suivie de quelques brèves notes esquissant un "monologue du père" et des "dialogues" entre le père et les différents membres de la famille. On se souvient que Le Retour de l'Enfant Prodigue est aussi composé d'une suite de dialogues. Suit un monologue où Profitendieu médite sur ses relations avec Bernard; il est trois fois interrompu par des clients, par Cécile et par Caloub; puis on sonne à la porte. Dans le roman, c'est Marguerite Profitendieu qui entre seule à ce moment, Profitendieu l'avertit de l'absence de Bernard et lui demande de venir le voir avec les enfants, après le dîner. Dans le manuscrit, d'abord entre un vieil ami de Profitendieu, suivi quelques minutes plus tard par Charles le fils aîné, enfin arrive Marguerite en compagnie d'une jeune amie. Une longue discussion s'engage à table jusqu'à ce que Profitendieu se sente mal; les invités se retirent; alors le manuscrit rejoint le texte définitif: la famille se réunit autour du père, Profitendieu donne une explication évasive de l'absence de Bernard et congédie les enfants; il fait lire la lettre de Bernard à Marguerite; Charles revient consoler son père; le chapitre se termine sur un épilogue montrant chacun dans sa chambre pendant que Bernard monte en tapinois l'escalier qui conduit à la chambre d'Olivier.

L'étude détaillée de ce manuscrit confrontée à certaines informations tirées du Journal des Faux-Monnayeurs et de la Correspondance Gide-Martin du Gard montre que Gide avait écrit ce texte sans faire de Bernard un bâtard. C'est seulement à la date du 7 octobre 1922 (il travaille sur les Faux-Monnayeurs depuis 1919) qu'il décide de faire de Bernard un enfant adultérin qui découvrant ce fait quitte la maison de son père putatif. On devine donc que la lettre explicative de Bernard date de cette décision et que le feuillet la contenant a été ajouté aux autres et l'ensemble a été remanié pour s'accorder à cette nouvelle donnée. Et c'est seulement au début de l'année 1924 (un an avant la parution du roman) que l'auteur décide de supprimer le long épisode de la discussion à table (une dizaine de feuillets) et de ressouder le reste pour l'amener à l'état définitif. 4 
Le fait le plus marquant dans cette transition est évidemment la suppression du long épisode que l'on peut baptiser l'épisode des "deux fâcheux" en empruntant l'expression au texte lui-même, et la réduction apportée au monologue de Profitendieu ainsi qu'au dialogue entre lui et Charles.

Structurellement, l'épisode des "deux fầcheux" est une comédie bourgeoise en une scène. Profitendieu est tenaillé par une crise hépatique déclenchée au choc que lui a causé la lettre de Bernard. Sa méditation vient d'être interrompue à trois reprises sous l'oeil narquois de son serviteur. Il attend impatiemment sa femme; mais voici qu'entre un premier "fâcheux" qui lui fait des compliments sur sa mine et l'engage à apprécier deux tableaux accrochés au mur. On apprend que c'est le "fâcheux" qui lui a conseillé de les acheter pour fin d'investissement lucratif. Arrive son fils aîné à qui il glisse furtivement un mot au sujet de la fugue de Bernard en le priant de se charger de l'intrus. Il est tout essoufflé de cette première manoeuvre qu'entre enfin son épouse, gaie et fringante à le faire sentir "tout vieilli." Elle présente une jeune amie qu'elle a "amenée dîner" dont elle ne sait pas exactement le nom (elle hésite entre Freyda, Hilda et Bronia van den Floup) et qui vient de passer trois ans toute seule parmi les sauvages en Poméranie, en Océanie ou aux îles Marquises, elle ne sait. Elle s'empêtre à tel point qu'elle finit par demander à cette demi-Hollandaise de se présenter elle-même au premier intrus, et c'est à peine si elle s'aperçoit que son mari est "tout chose." Ce dernier arrive enfin à placer un mot sur l'absence de Bernard. Antoine annonce le dîner. Alors s'engage la discussion entre les "deux fâcheux," l'un toujours complimenteur, l'autre indifférente mais grave. Madame Profitendieu s'évertue à y faire participer Charles et Cécile qui restent interdits devant les manières et les propos de Bronia, trop libres à leur goût. Profitendieu essaie de cacher son malaise devant l'assiette qu'il a remplie "pour ne pas attirer l'attention;" il finit par s'excuser "d'une voix étranglée" et se précipite dehors lorsque les paroles de Bronia touchent trop insupportablement à la situation dramatique qui précisément le torture.

Cette scène se caractérise par un grand effort vers le réalisme, par une intense charge ironique et par sa totale gratuité. Il suffit de simplement parcourir les feuillets pour s'apercevoir de l'intention qu'a eue Gide d'y apporter tous les "effets de réel" conventionnels: tandis que le discours péremptoire de Bronia rédigé sans aucune retouche répond aux questions insidieuses et insipides du premier "fâcheux," les didascalies comportent un grand nombre de ratures et d'ajouts. Dans ce débat auquel Madame Profitendieu s'efforce de donner le tour d'une conversation mondaine, chacun a ses tics de langage, son vocabulaire, et l'on hésite parfois entre le "tu" et le 
"vous." De longues indications scéniques décrivent et nuancent les gestes et les mouvements, les jeux de regards, les tons de voix, les modulations de phrases, les silences et les attaques, les rougissements et les pâleurs. A ces indications se mêle une forte dose de commentaires du narrateur, approbatifs s'il s'agit de Bronia, d'une ironie acerbe à l'égard des autres, et ce persiflage accompagne aussi bien les gestes des personnages que la substance de leurs répliques. Gide semble avoir écrit le passage dans une sorte de rage qu'il exprime directement par ses multiples interventions. Puis il y a le réalisme descriptif très rare chez Gide, non plus description de ce qui change, mais de ce qui est, du physique des personnages, de leurs vêtements, des formes et des couleurs, des matières et des textures. La personne de Bronia est décrite en détail, depuis "sa chevelure courte et opulente" jusqu'à "sa manière libre de croiser ses jambes" en passant par son "collier de minuscules coquilles couleur rose-thé qui s'harmonise si suavement avec les tons ambrés de sa gorge." Par contraste avec le portrait de Bronia, celui de Cécile est destiné à la rendre inexistante: "elle exagère, par réaction, sa réserve, baisse un peu plus les yeux et porte un peu plus droit la tête, raidit le buste et décolore sa voix [...] elle passe complètement inaperçue."

Or ni l'abondance du réalisme ni le mordant de l'ironie ne sauvent l'épisode de sa gratuité, et Gide le savait en l'écrivant; car malgré les tensions et les attractions qui remuent les personnages en présence, il intervient pour préciser ceci à propos de Cécile et de Bronia:

Mais il suffit de voir un instant ces deux jeunes filles pour comprendre qu'entre elles aucune sympathie n'est possible et que cette présentation "ne donnera rien" comme l'on dit en argot de romancier.

et un peu plus loin:

La seule personne avec qui Bronia pourrait espérer un écho c'est Bernard. Ces deux êtres étaient peut-être faits pour s'entendre; mais précisément quand l'un arrive, l'autre s'en va.

Pourtant, eût-il été vraiment impossible d'imaginer des interactions entre ces personnages? Dans l'univers fragmenté des Faux-Monnayeurs où les êtres évoluent apparemment comme des atomes aux mouvements aléatoires et agissent les uns sur les 
autres par de secrètes influences, eût-il été invraisemblable que Bernard rencontrât un jour Bronia, ou que Cécile se découvrît un goût pour les joies de l'existence nomade de la jeune Hollandaise? Déjà au cours de la scène même, Profitendieu ne sent-il pas se réveiller en sa femme "par affinité, par contact, un vieux fond de protestantisme, qu'il espérait [...] à tout jamais recouvert?" Charles est à tel point troublé par "l'obscure chaleur qu'il sent émaner d'elle et l'envahir" qu'il reconnaît "tout aussitôt en cette protestante un suppôt de l'Enfer" et qu'il se précipite d'appuyer ses lèvres sur sa "petite médaille de la Sallette" pour y chercher protection à sa virginité. Et le premier fâcheux, tout émoustillé, propose de raccompagner la jeune fille chez elle après le dîner; or le narrateur intervient sarcastiquement pour couper court à cette galante entreprise.

On ne peut s'empêcher de voir dans la gratuité de cet épisode un parti pris d'inconséquence narrative, comme cette "inconséquence des personnages" qui fascinait Gide lecteur de Dostoïevski, inconséquence dont Gide réclamait la conformité à la vie même dans son Journal des Faux-Monnayeurs:

La vie nous présente de toutes parts quantités d'amorces de drames mais il est rare que ceux-ci se poursuivent et se dessinent comme a coutume de les filer un romancier. Et c'est là précisément l'impression que je voudrais donner dans ce livre, et c'est ce que je ferai dire à Edouard (JFM 80).

Et il est vrai que le roman définitif comporte nombre de personnages épisodiques, de personnages dont on parle à peine ou qui se manifestent soudain et dont on ne soupçonnait pas l'existence. A l'instar du roman tout entier qui "pourrait continuer," l'épisode des "deux fâcheux" répondait au désir d'éparpiller l'intrigue, de faire oeuvre "inassimilable à rien d'autre."

Sur le plan des idées aussi, cet épisode était un des plus importants, car là se débattaient nombre de thèmes essentiels au roman et permanents dans la pensée de Gide: éloge du nomadisme, critique de la famille bourgeoise, refus de toute dépendance, l'exaltation dans le dénuement, ouverture vers les autres, recherche de l'authenticité, réalisation de soi, et jusqu'à la question de l'enfant naturel ou adopté qu'on aime mieux que les siens propres, thèmes dont on trouve quelques échos dans la lettre de Bernard et dans les réflexions de Profitendieu et qui rejoignaient ceux du Retour de 
l'Enfant Prodigue. La thématique de l'épisode réalisait parfaitement ce que Gide se proposait de faire dès 1919, "juxtaposer et imbriquer à la manière de César Franck, un motif d'andante et un motif d'allegro" ou ce qu'il appellera plus tard "l'art de la fugue."

Il convient de se demander donc pour quelle raison un personnage et un épisode qui répondaient si bien aux desseins esthétique et thématique de l'oeuvre en ont été écartés. Quelle autre exigence, plus impérieuse, a mis fin à l'existence de Bronia après une telle longévité? Que les thèmes exposés par elle soient finalement versés dans le journal d'Edouard et dans d'autres dialogues ne diminue en rien la légitimté de la question. Ce transfert ne constitue en fait qu'une preuve supplémentaire de la validité du personnage en tant que porteur de thèmes. Mais que ce rôle soit dévolu à d'autres personnages signifie bien que Bronia n'avait pas sa place dans le roman, et qu'elle pouvait encore moins incarner la thématique fondamentale de l'oeuvre.

Or l'élimination de Bronia est contemporaine de quelques autres modifications, et l'examen attentif des variantes montre à l'évidence qu'elles tendent toutes à modeler l'aventure de Bernard sur celle de l'Enfant Prodigue. Nous avons déjà constaté que, dans le texte définitif, la famille Profitendieu est structurée comme celle du Prodigue gidien. Il fallait aussi attribuer aux membres de cette famille des caractéristiques semblables à celles des membres de la famille du Prodigue. Et c'est pour répondre à cette exigence que toutes les modifications ont été apportées.

Dans l'épisode des "deux fâcheux," Marguerite s'imposait de toute sa présence et persistait à vouloir diriger la conversation. C'était une mondaine qui avait passé son après midi à rendre des visites et à courir les bijouteries dans l'une desquelles elle avait rencontré Bronia. Mère dominatrice, elle embarrassait Charles en dénonçant son incapacité à voyager et regrettait que Cécile ne fût pas aussi hardie que Bronia. On eût dit qu'elle était prête elle-même à prendre le large. Dans le roman définitif, bien que rebelle et adultère autrefois, elle s'est effacée, elle est refoulée au second plan et comme enveloppée dans une digne résignation.

Charles était peint comme un jeune homme bourgeonneux, timide et maladroit qui en présence de Bronia se sentait en proie à des émois que "Dieu ne lui avait jamais donné d'éprouver auprès de sa fiancée." Et Dieu lui-même se trouvait quelque peu malmené par le sarcasme envahissant du narrateur. 
Le personnage de Profitendieu était à la limite du burlesque. C'est aussi contre lui que s'exerçait le sarcasme le plus agressif, directement par les commentaires du narrateur, et indirectement par le discours de Bronia. Car tout ce que disait cette dernière visait et tourmentait Profitendieu sans qu'il eût la force ni l'occasion d'intervenir dans la discussion. Même l'attitude d'Antoine a été modifiée dans la version définitive de telle sorte que Profitendieu ne soit pas humilié par le valet. Son effort de recueillement déjà deux fois interrompu par ses enfants était totalement mis en échec par ce long épisode. Dans la version définitive, sa méditation se concentre en une seule séquence et reste empreinte d'une dignité tragique. Il ne s'inquiète pas de ce qu'on va lui servir à dîner, ses protestations de l'homme parfait dans l'exercice de la paternité ont disparu, sa douleur physique se confond avec sa tristesse, le ressentiment à l'égard de Bernard a fait place à l'affection. Il force plutôt le respect que l'ironie quand il reconnaît qu'il "aimait Bernard comme il n'avait jamais aimé" ses autres enfants précisément à cause de ce qu'il sentait en lui "de neuf, de rude et d'indompté." L'on sait que le père du Prodigue ne blâme point son fils de l'avoir abandonné. Il sait ce qui poussait le Prodigue sur les routes, et il l'attendait au bout comme Profitendieu est prêt à recevoir Bernard à la fin de son périple.

Enfin, il est vrai que le lecteur connaît peu Caloub. Mais à l'instar des classiques, Gide sait tracer un portrait moral à l'aide de quelques traits, ou situer un personnage dans un contexte par de rares notations. L'apparentement de Caloub au puîné de l'Enfant Prodigue est effectué par ce procédé de litote. De même que la mère du Prodigue gidien s'inquiète des lectures de son dernier né, de même Profitendieu veille aux lectures de Caloub.

Ce qui semble en travail dans ce chapitre II du roman est la la version gidienne de la parabole évangélique qui comme un schéma dynamique tend à produire non pas une nouvelle version du "retour" du Prodigue, mais les circonstances de son départ, et à peindre, au delà de ce chapitre, son aventure dans le vaste monde, son combat avec l'ange et la découverte de ses possibles. Le traité de 1907 et le roman de 1925 se rejoignent lorsque prend fin l'aventure du fils prodigue. La phrase finale du roman suggère symboliquement le départ de Caloub, comme la dernièe phrase du traité montre le Prodigue aidant son jeune frère à partir. Le parallélisme textuel va jusqu'à produire deux épilogues symétriques dans la nuit qui tombe après le retour de l'Enfant Prodigue et après le départ de Bernard: 
A présent, dans la nuit où pas un souffle ne s'élève, la maison fatiguée âme après âme, va s'endormir. Mais pourtant, dans la chambre à côté de celle du prodigue, je sais un enfant, son frère cadet, qui toute la nuit jusqu'à l'aube va chercher en vain le sommeil (Le Retour de l'Enfant Prodigue, Pléiade, 477).

Le père et le fils n'ont plus rien à se dire. Quittons-les [...] Laissons madame Profitendieu dans sa chambre, [...] Elle ne pleure pas; elle ne pense à rien [...] Quittons-la. Cécile dort déjà. Caloub considère avec désespoir sa bougie; elle ne durera pas assez pour lui permettre d'achever un livre d'aventures, qui le distrait du départ de Bernard (Les Faux-Monnayeurs, Pléiade, 950).

Le rejet de l'épisode des "deux fâcheux" était donc inscrit dans les structures signifiantes du mythe qui ordonnait le chapitre. Dans ce tableau de famille, les intrus ne pouvaient être admis. Bronia aurait pu incarner à sa manière le mythe de l'Enfant Prodigue, mais pas dans Les Faux-Monnayeurs. Gide lui a pourtant réservé une place, elle aura "voix au chapitre," métamorphosée en Geneviève, dans la trilogie de L'École des femmes. Comme Bronia du manuscrit, Geneviève sait infiniment mieux parler que Bernard; mais elle ne parle pas d'expérience et elle désavoue ses "belles théories"5 sans les avoir mises en pratique. La passivité de Geneviève est peut-être signe que la psyché gidienne n'a jamais pu concevoir d'enfant prodigue qu'au masculin. 


\section{Notes}

1 Voir Un Fragment des Faux-Monnayeurs, le manuscrit de Londres présenté par N. David Keypour, Centre d'Études Gidiennes, Lyon, 1990.

2 Bien que le titre de cet article évoque les analyses de Northrope Frye dans son Anatomie de la critique, l'approche s'apparente plutôt à celle de Charles Mauron exposée dans Des métaphores obsédantes au mythe personnel. Cependant, le procéessus ici employé diffère de celui de Mauron en ce que le mythe personnel de Gide ne s'établit pas à partir d'un rapprochement d'images recueillies dans l'ensemble de son oeuvre, mais à partir de l'interprétation personnelle que Gide donne d'un mythe déjà existant. C'est pourquoi, l'expression "mythe personnel" n'implique ici aucun parti pris pour ou contre le rôle de l'inconscient dans la théorie psychocritique de Mauron. Autrement dit, l'adjectif "personnel" est employé comme un simple possessif, sans aucune charge psychologique. C'est que le cadre restreint de cette étude ne permet pas d'appliquer l'analyse à d'autres écrits Gidiens. Il y aurait certainement lieu de procéder avec l'oeuvre de Gide comme Mauron l'a fait pour Racine: établir d'une manière détaillée toutes les dimensions narratives et thématiques du mythe gidien de l'Enfant Prodigue, en suivre la transformation d'oeuvre en oeuvre, et voir ensuite si ce mythe peut être tenu pour l'image de la personnalité inconsciente de l'auteur.

3 Il n'échappe pas à l'attention que la structure de cette famille diffère de celle de la famille archétypale aussi par la fait qu'elle comporte des soeurs. Il en va de même de la famille Profitendieu qui est pourtant la plus conforme au modèle et qui fera l'objet particulier de cette étude. Mais il semble bien que dans la constitution de son mythe personnel, Gide néglige les soeurs, on pourrait même affirmer sans risque de se tromper qu'il les tient pour des quantités négligeables s'il n'était pas vrai qu'elles représentent dans certaines familles, comme il est le cas ici, la négativité contagieuse, la mollesse, le marécage de Paludes qui est "spécialement l'histoire de qui ne peut pas voyager," de l'homme "qui ne s'efforce pas d'en sortir" (Pléiade, 91). Ces remarques sont également à considérer en lisant l'orientation de critique féministe suggérée en conclusion de cette étude. 
4 Sur tous ces points, voir l'introduction à Un fragment des Faux-Monnayeurs, 16-18.

5 C'est l'expression utilisée par Profitendieu pour se moquer des idées de Bronia; Marchant de L'École des femmes l'emploie pour qualifier les idées de Geneviève. 